\title{
Bus Load Decomposition Method Based on Deep Learning
}

\author{
Tiantian Qian ${ }^{1,}$, , ke Wang ${ }^{1}$, and Fei $\mathrm{Shi}^{1}$, Gang Li $^{2}$, Lizhong $X u^{3}$ \\ ${ }^{1}$ China Electric Power Research Institute Co., Ltd., 210000 Nanjing, China \\ ${ }^{2}$ State Grid Jiangsu Electric Power Co., Ltd., 210000 Nanjing, China \\ ${ }^{3}$ State Grid Zhejiang Electric Power Co., Ltd., 310000 Hangzhou, China
}

\begin{abstract}
The current research work is mainly based on the decomposition of the total load of the family house into the electrical level load, and less research on the bus load of the high voltage level. To solve this problem, in this paper, a bus load composition decomposition algorithm based on Bi-directional Long Short-Term Memory (Bi-LSTM) is proposed. The experimental results show that this method can effectively identify the bus load with unknown components. Compared with the traditional recurrent neural network and long-term and short-term memory network, the proposed algorithm has better identification ability.
\end{abstract}

\section{Introduction}

Some buses of medium and high voltage level in power grid, such as $220 \mathrm{kV}$ buses of provincial power grid and $110 \mathrm{kV}$ buses of regional power grid, etc, which usually converge a large number of load resources with different types and characteristics. How to effectively predict the power changes of these bus loads and how to effectively utilize the flexible loads gathered on these buses are the key concerns of power dispatchers [1-4].

Identifying and decomposing bus loads to obtain the types and quantities of bus loads is the basis and key to carry out the above research. However, there are few studies on load decomposition of medium and high voltage buses at home and abroad.

In this paper, the terminal load of medium and high voltage bus load is defined as the power load of building groups, such as schools, shopping malls, hotels, hospitals, etc. Because the electricity load of different types of building groups has its obvious characteristics of electricity consumption [5]. The problem that the identification of bus load composition to be solved is that, for a given building group load feature library and the terminal load features extracted from the measured data, it can identify which terminal loads are included in the bus load and how much the bus load is, in order to realize bus load decomposition. Mathematical optimization and pattern recognition are two kinds of solving methods for load identification and decomposition problems [6,7]. In recent years, with the development of artificial intelligence technology, the problem of load decomposition in pattern recognition has gradually attracted wide attention of scholars at home and abroad [8-9]. The research idea of pattern recognition is to learn a function (parameters of neural network model) from a given training data set of bus load decomposition recognition. When there are new unknown components in bus load data set, the decomposition results can be predicted based on this function.

In summary, aiming at the problem of load identification of medium and high voltage bus, this paper takes advantage of artificial intelligence to deal with complex data relations with non-linearity and interaction. A bus load decomposition identification algorithm based on Bi-directional Long Short-Term Memory (LSTM) is proposed. The experimental results show that it can effectively identify and decompose the load types and loads contained in medium and high voltage class buses.

\section{Bus Load Decomposition Method}

The problem of bus load decomposition is described as follows.

Target Tasks: the bus load change sequence, $X=\left\{x_{1}, x_{2}, \cdots, x_{\mathrm{T}}\right\}, x_{t}$ is the bus load value in $t=\{1,2, \cdots, \mathrm{T}\}, x_{t}$ is the composite of $\mathrm{N}$ types of terminal loads, such as the load of apartment complex, shopping mall complex, school complex, etc. The objective of load identification is to estimate the load variation sequence of each type of terminal load $Y^{i}=\left\{\mathrm{y}_{1}^{i}, \mathrm{y}_{2}^{i}, \cdots, \mathrm{y}_{T}^{i}\right\}$. Make its load value at time $\mathrm{t}$ satisfy the equation (1).

$$
x_{t}=\Sigma_{i=1}^{N} y_{t}^{i}+\sigma(\mathrm{t})
$$

In equation (1), $\sigma(\mathrm{t})$ represents other types of terminal load that are not considered and the influence of noise.

Learning tasks: given training set $\left\{\left(\mathrm{X}_{1}, \mathrm{Y}_{1}\right),\left(\mathrm{X}_{2}, \mathrm{Y}_{2}\right), \cdots,\left(\mathrm{X}_{n}, \mathrm{Y}_{n}\right)\right\}$ and verification set

\footnotetext{
* Corresponding author: qiantiantian@epri.sgcc.com.cn
} 
$\left\{\mathrm{X}_{\mathrm{n}+1}, \mathrm{X}_{\mathrm{n}+2}, \cdots, \mathrm{X}_{\mathrm{n}+\mathrm{m}}\right\}$, in which $\left(\mathrm{X}_{\mathrm{k}}, \mathrm{Y}_{\mathrm{k}}\right)$ is the k-th training sample, $k=\{1,2, \cdots, \mathrm{n}\}$.

$\mathrm{X}_{\mathrm{k}}$ is the sequence of bus load change. $\mathrm{Y}$ is the sequence of load changes corresponding to $\mathrm{N}$ terminal loads, $\mathrm{Y}_{\mathrm{k}}=\left\{\mathrm{Y}_{\mathrm{k}}^{1}, \mathrm{Y}_{\mathrm{k}}^{2}, \cdots, \mathrm{Y}_{\mathrm{k}}^{\mathrm{N}}\right\}$. Based on the training set, the learning model $\mathrm{M}$ can be trained. $\mathrm{M}$ can be used to predict the corresponding terminal load change sequence $Y_{j}=\left\{Y_{j}^{1}, Y_{j}^{2}, \cdots, Y_{j}^{N}\right\}$ for each $X_{j}, j=\{n+1, n+2, \cdots, n+m\}$ on the test set.

The detailed research flow of the bus load decomposition method proposed in this paper is shown in Fig. 2. The specific steps are as follows:

Step 1: Collect the standard annual electricity consumption data of typical building load.

Step 2: Collect external multi-source information, such as external meteorology (such as temperature, humidity, wind direction, wind speed and solar radiation heat) and date types (holidays, working days, nonworking days) that affect the change of building electricity load in the corresponding standard year.

Step 3: Generate training set and verification set for training and validating network model parameters, then train two-way long-term and short-term memory network with training set samples, and validate the validity of network model parameters generated by training link with validation set.

Step 4: Based on the trained two-way long-term and short-term memory network, the terminal load type and number of bus loads with unknown components are decomposed and identified.

The processing method for input sequential data is as follows:

Normalization: Min-max is used for normalization.

$$
x^{*}=\left(x-x_{\min }\right) /\left(x_{\max }-x_{\min }\right)
$$

In the equation (2), $x_{\max }$ is the maximum of the sample data and $x_{\min }$ is the minimum of the sample data.

Filtering: The input time series data are processed by uniform quantization, that is to say, the average interval of the input signal range is divided to eliminate the instantaneous peak value.

$$
Q_{y}(t)=\delta \cdot \text { floor }\left[\frac{P_{y}(t)}{\delta}+\frac{1}{2}\right]
$$

In the equation (3), floor (x) function is a downward integral function, $P_{y}(t)$ is the total power time series, $\delta$ is the quantized parameter, and $Q_{y}(t)$ is the quantized time series.

Differential processing: The total power value of adjacent time points after filtering is subtracted to obtain the differential waveform of total power.

$$
d t f f(t)=Q_{y}(t)-Q_{y}(t-1)
$$

The hardware configuration of the machine used in this experiment is Intel (R) Core (TM) i7-6700 CPU@ 3.4 GHz. The program compiler is Python 3.5. The artificial intelligence algorithm framework is based on TensorFlow, which is the second-generation artificial intelligence learning system developed by Google.

The experimental data are based on EnergyPlus software to generate five typical building load curves for one year. Thirty different bus loads are synthesized by multiplying different coefficients. The external information source data used are date type (workday or holiday), temperature. The input of network model: date type, temperature, total bus load, bus load differential quantity. The label is: each building load electricity consumption; output quantity is: each building load forecast value. In this paper, the annual load curve is divided into 1440 groups of data in weekly units. The first 1296 sets of data are used as training sets, and the last 144 sets of data are used as validation sets. The data of one week in the verification set is selected as representative, and the result of load decomposition of a bus is shown in Figure 1.

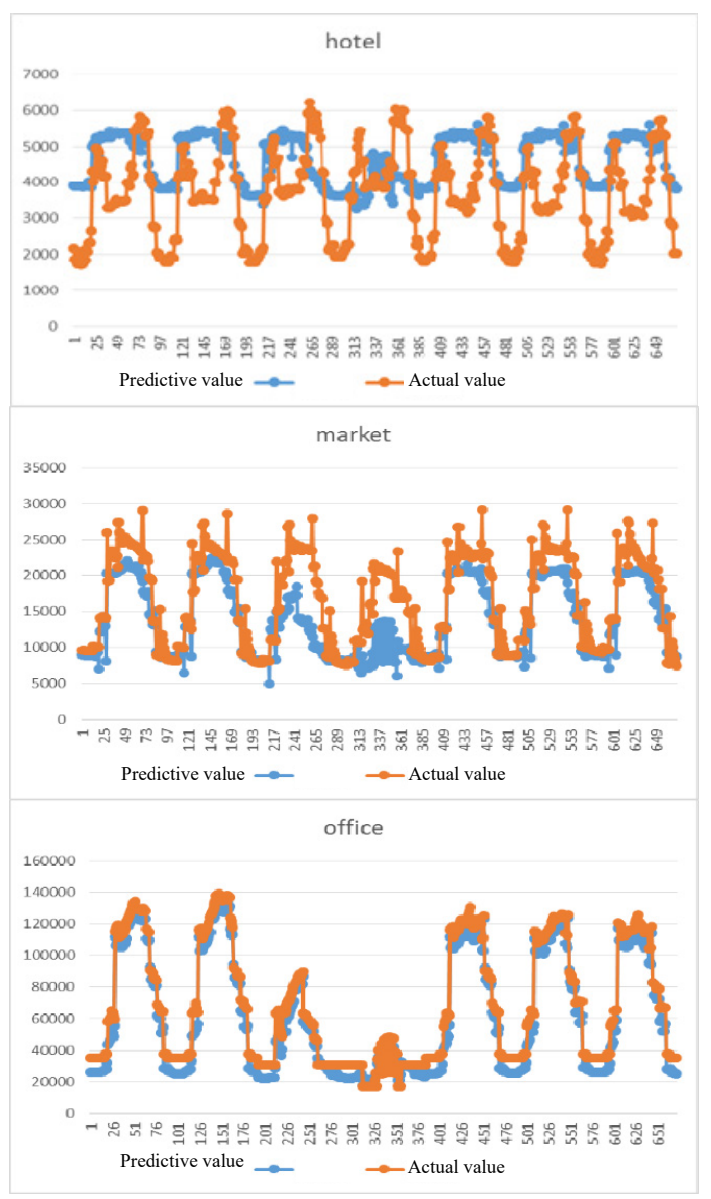

\section{Case Verification of Bus Load Decomposition}




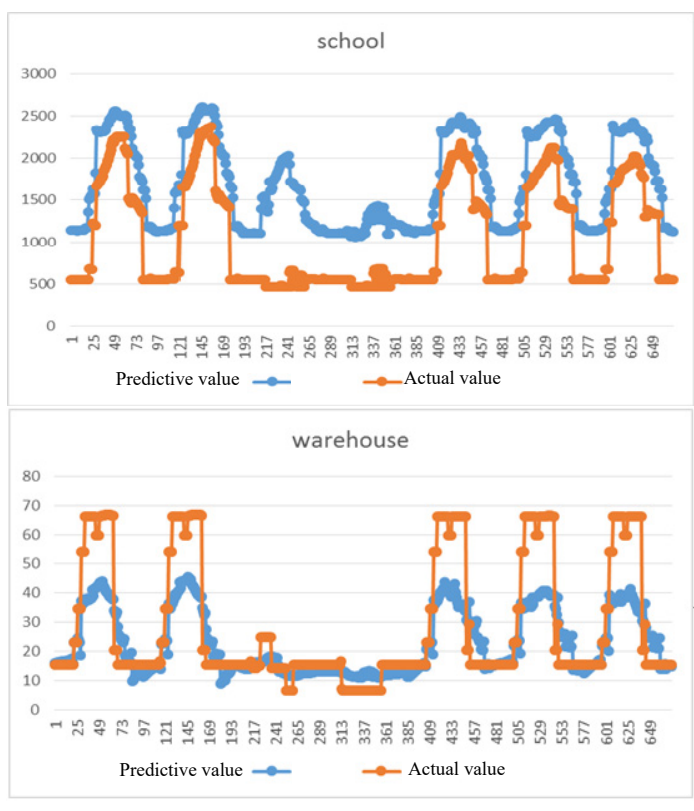

Fig. 1. The result of bus load decomposition identification (comparison of predicted and actual values)

In order to quantitatively evaluate the identification effect, the mean relative error (MRE) is used as an evaluation index to evaluate the errors of predicted and real values at all times. The calculation formula is shown in equation (5).

$$
\operatorname{MRE}=\frac{1}{\mathrm{~T}} \sum_{\mathrm{t}=1}^{\mathrm{T}} \frac{\left|\hat{p}_{\mathrm{t}}-p_{\mathrm{t}}\right|}{p_{\mathrm{t}}}
$$

In equation (5), $p_{\mathrm{t}}$ is the real power of a building load under the bus load at time $\mathrm{t}, \hat{p}_{\mathrm{t}}$ is the predicted power of a building load under the bus load at time $t$, and $\mathrm{T}$ is an artificial period of time, where $\mathrm{T}$ takes a week.

In Figure 3, the blue curve is the predicted value and the red curve is the real value. The average relative error between the real value and the predicted value is shown in the results under parameter settings 2 in Table 1. Parameter setting refers to the setting of some key training parameters (such as time step, number of hidden layer nodes, sliding step, etc.) in the process of network training. The results of bus load decomposition identification with different parameter settings and different proportion of building load are shown in Tables 1 and 2. It can be observed that, (1) These training parameters have a great influence on the accuracy of identification results. In the course of training, it is necessary to adjust these parameter settings many times in order to obtain the optimal identification results. (2) The higher the proportion of building load in total load is, the lower the identification error is.

Table 1. Case 1, the bus load decomposition result under different parameter settings (the average relative error between the target value and the predicted value)

\begin{tabular}{|c|c|c|c|c|c|}
\hline $\begin{array}{c}\text { The } \\
\text { proportion } \\
\text { of building } \\
\text { loads in } \\
\text { total buses }\end{array}$ & $4 \%$ & $17 \%$ & $61 \%$ & $1 \%$ & $17 \%$ \\
\hline $\begin{array}{c}\text { Parametric } \\
\text { Settings I }\end{array}$ & $33.04 \%$ & $18.29 \%$ & $17.54 \%$ & $66.38 \%$ & $41.77 \%$ \\
\hline
\end{tabular}

\begin{tabular}{|c|c|c|c|c|c|}
\hline $\begin{array}{c}\text { Parametric } \\
\text { Settings II }\end{array}$ & $27.94 \%$ & $18.90 \%$ & $18.64 \%$ & $39.36 \%$ & $46.23 \%$ \\
\hline $\begin{array}{c}\text { Parameter } \\
\text { Settings III }\end{array}$ & $34.95 \%$ & $30.80 \%$ & $18.59 \%$ & $44.46 \%$ & $34.32 \%$ \\
\hline $\begin{array}{l}\text { Parametric } \\
\text { Settings IV }\end{array}$ & $30.36 \%$ & $22.89 \%$ & $17.78 \%$ & $57.42 \%$ & $25.04 \%$ \\
\hline $\begin{array}{l}\text { Parametric } \\
\text { Settings V }\end{array}$ & $40.78 \%$ & $19.77 \%$ & $13.85 \%$ & $98.07 \%$ & $28.18 \%$ \\
\hline
\end{tabular}

Table 2. Case 2, the bus load decomposition result under different parameter settings (the average relative error between the target value and the predicted value)

\begin{tabular}{|c|c|c|c|c|c|}
\hline $\begin{array}{c}\text { The } \\
\text { proportion } \\
\text { of building } \\
\text { loads in } \\
\text { total buses }\end{array}$ & $5.01 \%$ & $21.04 \%$ & $72.81 \%$ & $1.12 \%$ & $0.03 \%$ \\
\cline { 2 - 6 } & hotel & supermarket & $\begin{array}{c}\text { office } \\
\text { building }\end{array}$ & school & warehouse \\
\hline $\begin{array}{c}\text { Parametric } \\
\text { Settings I }\end{array}$ & $33.04 \%$ & $18.29 \%$ & $17.54 \%$ & $66.38 \%$ & $41.77 \%$ \\
\hline $\begin{array}{c}\text { Parametric } \\
\text { Settings II }\end{array}$ & $27.94 \%$ & $18.90 \%$ & $18.64 \%$ & $39.36 \%$ & $46.23 \%$ \\
\hline $\begin{array}{c}\text { Parameter } \\
\text { Settings III }\end{array}$ & $34.95 \%$ & $30.80 \%$ & $18.59 \%$ & $44.46 \%$ & $34.32 \%$ \\
\hline $\begin{array}{c}\text { Parametric } \\
\text { Settings IV }\end{array}$ & $30.36 \%$ & $22.89 \%$ & $17.78 \%$ & $57.42 \%$ & $38.14 \%$ \\
\hline $\begin{array}{c}\text { Parametric } \\
\text { Settings V }\end{array}$ & $40.78 \%$ & $17.11 \%$ & $25.78 \%$ & $42.26 \%$ & $42.97 \%$ \\
\hline
\end{tabular}

It can be concluded from the above analysis that the proposed bus load decomposition identification method is feasible. The bus load with unknown component can be decomposed into terminal load with building load as unit accurately, and the decomposition error can be controlled within a certain range.

\section{Conclusion}

The existing load identification and decomposition methods are mostly aimed at small-scale household users. The research methods are difficult to apply to large-scale and diversified load side resource decomposition and identification problems of medium and high voltage bus. The bus load identification decomposition method based on bidirectional long-term and short-term memory network proposed in this paper can effectively identify the types and loads of terminal buildings contained in decomposition buses of medium and high voltage grades. It can provide a powerful analysis basis for subsequent bus load forecasting and demand response management. However, the identification accuracy of building load, which accounts for a small proportion of bus load, needs to be further improved. The next work will focus on this issue.

\section{Acknowledgement}

The authors gratefully acknowledge the sponsor of the project of Research on Intelligent Identification and Short-term Forecasting Method of Regional Bus Load Based on Deep Learning (52420018004W) and the State Grid Corporation of China Science Project (52110418002A) and State Grid Jiangsu Provincial Electric Power Co., Ltd. Science and Technology Project "load recovery strategy research in later period of UHVDC fault dispose". 


\section{References}

1. LI Bo, MEN Deyue, YAN Yaqin, etal. Bus load forecasting based on numerical weather prediction $[\mathrm{J}]$ Automation of Electric Power Systems, 2015, 39(1): 137-140.

2. ZHU Zelei, CHENG Xin, YANG Guizhong, etal. Collaborative scheduling optimization method for provincial grid and regional grid based on refined busload forecast $[\mathrm{J}]$. .Proceedings of the CSEE, 2017, 37( 3): $665-676$.

3. CHENG Lin1, WAN Yuxiang1, ZHANG Fang1, etal. Operation Mode and Control Strategy for Airconditioning Service Based on Business of Load Aggregator[J]. Automation of Electric Power Systems, 2018, 42(18):8-18.

4. Luo Bofeng, Mu Yunfei, JiaHongjie, et al Decision method of power supply access for large consumers based on load feature library[J]. Automation of Electric Power Systems, 2018, 42(6): 66-72.

5. Xiao F, Fan C. Data mining in building automation system for improving building operational performance[J]. Energy and buildings, 2014, 75: 109-118.

6. LI Peng. Non-intrusive method for power load disaggregation and monitoring[D]. Tianjin: Tianjin University, 2009.

7. Srinivasan D, Ng W S, Liew A C. Neural-networkbased signature recognition for harmonic source identification [J]. IEEE Transactions on Power Delivery, 2006, 21(1):398-405.

8. WANG Ke, ZHONG Haiwang, YU Nanpeng, etal. Nonintrusive Load Monitoring based on Sequenceto-sequence Model With Attention Mechanism[J]. Proceedings of the CSEE, 2019, 39(1): 79-84.

9. YAN Xufeng, ZHAI Shaopeng, WANG Zhihua, etal. Application of Deep Neural Network in Nonintrusive Load Disaggregati[J]. Automation of Electric Power Systems, 2019, 43(1): 126-136. DOI: 10.7500 /AEPS20180629004. 